\title{
Some Phytochemicals Found in Medicinal Plants Used in Cancer - A Review
}

Vimla Kumari*, Kamini Kaushal, Ashwini K Sharma, Rajesh Chandra Mishra and Pradeep Soni

Department of Dravyaguna, Madan Mohan Malviya Government Ayurvedic College, Udaipur, Rajasthan, India

\begin{abstract}
In the last few decades, phytochemicals have potentially gained as an important place in cancer research. The interests for these compounds have grown by researchers, as these compounds have natural in origin and hence, no stipulated side effects are known. Currently, thousands of natural herbs based compounds have been screened for their novel efficiency to control cancer cell proliferation. Among these, a large number of natural compounds gained high preventive and therapeutic values against cancer and most potential compounds along of the are lupeol, saponins, flavanoids, curcumin, resveratrol, geneistein, gingerol, allyl sulfide, berberine, lycopene, bromelain, indole-3-carbinol and polyphenols. The recent research on phytochemicals towards evaluating the anticancer efficacy has been accelerated by development of biophysical technologies. However, several challenges still persist in cancer research like heterogeneity, development of novel phenotypes in cancer cell by therapeutic drugs and tumour recurrence. These challenges open a new dimension for cancer research, and also give weightage to explore the uses of specific phytochemicals in therapy. This review summarizes the latest research in cancer treatment using the bioactive components from medicinal plants.
\end{abstract}

Keywords: Phytochemical; Medicinal plants; Cancer; Cell proliferation

\section{Introduction}

Phytochemicals, also known as phytonutrients, are natural nonessential chemical compounds found in plants (phyto is a Greek word meaning "plant"). They can occur in vegetables, grains, legumes, beans, fruits, herbs, nuts, roots, leaves and seeds. Phytochemicals are compounds that give plants their color, flavor, and smell. These compounds are thought to be largely responsible for the medicinal properties and health benefits of medicinal herbs. Some protect against cancer when isolated, some are not associated with cancer at all, and many have yet to be discovered. It is known that many phytochemicals, when kept in their natural food forms, can protect us from cancer as they interact with other phytochemicals and the cells in our bodies. This chemical acts as an antioxidant similar to vitamins A, C, and E, and may help protect the body from free radicals. It is also possible that phytochemicals fights cancer by reacting with carcinogens and changing their structure so they can no longer initiate tumors or by speeding the death of cancer cells that have already formed [1-6].

Cancer is one of the most severe health problems in both developing and developed countries, worldwide. Among the most common (lung, stomach, colorectal, liver, breast) types of cancers, lung cancer has continued to be the most common cancer diagnosed in men and breast cancer is the most common cancer diagnosed in women. The International Agency for Research on Cancer estimates of the incidence of mortality and prevalence from major types of cancer, at national level, for 184 countries of the world revealed that there were 14.1 million new cancer cases, 8.2 million cancer deaths, and 32.6 million people living with cancer (within 5 years of diagnosis) in 2012 worldwide. By 2030, it is projected that there will be 26 million new cancer cases and 17 million cancer deaths per year [7-13].

Cancer remains to be one of the leading causes of death in the United States and around the world. The advent of modern drugtargeted therapies has undeniably improved cancer patients' cares. However, advanced metastasized cancer remains untreatable.

\section{Functions of Phytochemicals}

- Stimulate the immune system.

- Block substances we eat, drink and breathe from becoming carcinogens.

- Reduce the kind of inflammation that makes cancer growth more likely.

- Prevent DNA damage and help with DNA repair.

- Reduce the kind of oxidative damage to cells that can spark cancer.

- Slow the growth rate of cancer cells.

- Trigger damaged cells to commit suicide before they can reproduce.

- Help to regulate hormones.

Medicinal plants have been always an important source for the discovery of new therapeutics for human diseases. These sources may be a good candidate for the development of novel anticancer agents. Medicinal plants have been studied for cancer, only a small number of them pass in vitro experiments and animal studies and are under clinical trials. Based our literature search, lupeol, saponins, flavanoids, curcumin, resveratrol, geneistein, gingerol, allyl sulfide, berberine, lycopene, bromelain, indole-3-carbinol had satisfactory instances of clinical evidence for supporting their anticancer effects (Table 1). Therefore, it seems that they can be used as a complementary

*Corresponding author: Dr. Vimla Kumari, Department of Dravyaguna, Madan Mohan Malviya Government Ayurvedic College, Udaipur, Rajasthan, India, Tel: 0636865749 E-mail: kumarivimla1990@gmail.com

Received January 27, 2018; Accepted February 01, 2018; Published February 07, 2018

Citation: Kumari V, Kaushal K, Sharma AK, Mishra RC, Soni P (2018) Some Phytochemicals Found in Medicinal Plants Used in Cancer - A Review. Med Chem (Los Angeles) 8: 018-020. doi: 10.4172/2161-0444.1000491

Copyright: ( 2018 Kumari V, et al. This is an open-access article distributed under the terms of the Creative Commons Attribution License, which permits unrestricted use, distribution, and reproduction in any medium, provided the original author and source are credited. 
Citation: Kumari V, Kaushal K, Sharma AK, Mishra RC, Soni P (2018) Some Phytochemicals Found in Medicinal Plants Used in Cancer - A Review. Med Chem (Los Angeles) 8: 018-020. doi: 10.4172/2161-0444.1000491

\begin{tabular}{|c|c|c|c|}
\hline S No & Phytochemical & Pharmacological Activity & Effect in Cancers \\
\hline 1 & $\begin{array}{l}\text { Lupeol/ triterpene/ } \\
\text { Fagarsterol }\end{array}$ & $\begin{array}{c}\text { Anti-inflammatory, Anti-proliferative, Anti-tumour, Chemo preventive, Anti-microbial, } \\
\text { Anti-invasive }\end{array}$ & $\begin{array}{l}\text { Tumorigenicity of Prostate, skin, pancreatic, } \\
\text { head and neck squamous cell carcinoma }\end{array}$ \\
\hline 2 & Saponin & $\begin{array}{l}\text { Anti-hyperlipidemic Anti-inflammatory, expectorant, immune stimulating activity, anti- } \\
\text { cancer, anti-oxidative, anti-diabbetic, hepato-protective }\end{array}$ & Colon, lung cancer \\
\hline 3 & \multicolumn{3}{|c|}{ Phytosterols } \\
\hline A & Stigmasterol & $\begin{array}{l}\text { Anti-hyper cholestrolemic, Cytotoxicity, Anti-osteoarthritic, Hypoglycemic, Anti- } \\
\text { tumour, Anti-inflammatory }\end{array}$ & Skin cancer \\
\hline B & Campsterol & $\begin{array}{l}\text { Anti-tumour, Anti-osteoarthritic, Hypoglycemic, Antioxidant, Anti-inflammatory, Anti- } \\
\text { hypercholestromic }\end{array}$ & Breast, colon, lung cancer \\
\hline C & Beta-sitosterol & $\begin{array}{l}\text { Immunomodulatory effects, Anticancer, anti-atherogenic, reduced symptoms of } \\
\text { enlarged prostate }\end{array}$ & Prostate cancer \\
\hline 4 & \multicolumn{3}{|c|}{ Flavanoids } \\
\hline A & $\begin{array}{l}\text { Flavanoids- flavone, } \\
\text { Isoflavanoids }\end{array}$ & $\begin{array}{l}\text { inhibit inflammation and tumor growth; may aid immunity and boost production of } \\
\text { detoxifying enzymes in the body }\end{array}$ & $\begin{array}{l}\text { Human oral, rectal, prostate, Lung, leukemia, } \\
\text { stomach, thyroid, colon, laryngeal, breast, } \\
\text { Colorectal, kidney }\end{array}$ \\
\hline B & Luteolin & $\begin{array}{c}\text { Anti-oxidative, anti-inflammatory, Anti-microbial, Anti-tumour, inhibition of cell } \\
\text { proliferation, metastasis and angiogenesis }\end{array}$ & Breast, gastric, prostate cancer, \\
\hline C & Genistein & Estrogenic effect, Antioxidant, Anticancer, cardioprotective & $\begin{array}{l}\text { Leukemia, lymphoma, ovarian, cervical, } \\
\text { gastric, pancreatic, breast, prostate }\end{array}$ \\
\hline D & Quercetin & $\begin{array}{c}\text { inhibits cell proliferation Anti-inflammatory, analgesic, anti-allergic, anti- cancer, } \\
\text { chemopreventive, anti-oxidative }\end{array}$ & Breast cancer \\
\hline E & Epigallo-catechin gallate & Anticancer, chemopreventive, Antiproliferative & $\begin{array}{l}\text { Brain, prostate, cervical, bladder, colorectal } \\
\text { cancer }\end{array}$ \\
\hline 5 & Piperine & Anti-inflammatory, anti-tumour, & Breast, prostate cancer \\
\hline 6 & Diallyl sulphide & $\begin{array}{l}\text { Anti-proliferative, preventing bone loss, Anti-cancer, hepatoprotective, immune } \\
\text { stimulating, Anti-oxidative, Anti-hypercholestromic, Anticoagalative, detoxification, } \\
\text { antibacterial }\end{array}$ & Skin cancer \\
\hline 7 & Gingerol & Anti-oxadative, anti-inflammatory, anti-tumourogenic & Colon, breast, ovarian pancreas \\
\hline 8 & Crocetin & Inhibited cancer cell proliferation, antioxidant effects, chemopreventive & $\begin{array}{l}\text { Liver lung, pancreatic, skin, colorectal, breast } \\
\text { cancer }\end{array}$ \\
\hline 9 & $\begin{array}{c}\text { Curcumin } \\
\text { (Diferuloylmethane) }\end{array}$ & $\begin{array}{l}\text { Induce apoptosis in cancer cells without cytotoxic effects on healthy cells, Anti- } \\
\text { inflammatory, Antioxidant }\end{array}$ & $\begin{array}{l}\text { Colon, breast, lung metastases, brain tumor, } \\
\text { liver, leukemia }\end{array}$ \\
\hline 10 & $\begin{array}{c}\text { Indole-3-carbinol } \\
\text { (natural anti-carcinogen) }\end{array}$ & $\begin{array}{c}\text { Supports healthy estrogen metabolism, Improve hormone balance and fat burning, } \\
\text { Promotes liver detoxification, prevent cancer }\end{array}$ & Stomach, breast, colon, cervical, endometrial \\
\hline 11 & Lycopene & Preventing cancer and heart disease, Antioxidant & Prostate, breast, endometrial, colon \\
\hline 12 & Rosmarinic acid & Anti-oxidant and anti-inflammatory effects, protect against various forms of cancers & Colon, skin cancer \\
\hline 13 & Fisetin & Chemoprevention of cancer, anti-inflammatory, Antioxidant, Anti-carcinogenic & Colon cancer \\
\hline
\end{tabular}

Table 1: Anti-cancerous phytochemical and its activity.

\begin{tabular}{|c|c|c|}
\hline S No & Phytochemical & Medicinal Plants \\
\hline 1 & $\begin{array}{l}\text { Lupeol/triterpene (also known as } \\
\text { Fagarsterol) }\end{array}$ & $\begin{array}{c}\text { Amorphophallus campanulatus, Ficus lacor, Betula utilis, Jasminium auriculatum, Mimusops elengi, Bluea lacera, } \\
\text { Pterocarpus marsupeum, Diospyros tomentosa, Schleichera trijuga, Ricinus comunis, Soymida febrifuga, Grewia asiatica, } \\
\text { Ficus racemosa, Holorrhena antidycentrica, Crataeva nurvala, Salmalia malabarica, Bauhinia purpurea, Emblica officinalis, } \\
\text { Benincasa hispida, Wringtia tinctoria }\end{array}$ \\
\hline 2 & Saponin & $\begin{array}{l}\text { Asparagus adscendens, Curculigo orchioides, Costus speciosus, Mallotus philippensis, Madhuca longifolia, Trigonella } \\
\text { foenumgreacum, Luffa echinata, Sapindus trifoliatus, Althaea officinalis, Nigella sativa, Achyranthus aspera, Crataeva } \\
\text { nurvala, Nigella sativa, Hemidesmus indicus, Randia spinosa, Scirpus kysoor, Citrullus colocynthis, Pongamia pinnata, } \\
\text { Alternanthera sessilis, Callicarpa macrophylla, Cajanus cajan, Clerodendrum serratum, Coccinia indica, Luffa acutangula, } \\
\text { Phaseolus radiates, Borringtonia acutangula, Ficus hispida, Vernonia cineria, Streblus asper, Bombax ceiba, Albizzia } \\
\text { lebbeck, Lagenaria siceraria, Tamarindus indica, Musa paradisiacal, Centella asiatica, Caesalpinia sappan, Buchanania } \\
\text { lazan,, Asparagus racemosa, Dioscorea bulbifera, Acacia sumo, Careya arborea, Costus speciosus, Caesalpinia bonduc, } \\
\text { Smilex china, Gymnema sylvestre, Caesalpinia crista, Trichosanthes bracteata, tribulus terrestris }\end{array}$ \\
\hline 3 & & Phytosterols \\
\hline A & $\begin{array}{l}\text { Stimgasterol-an unsaturated } \\
\text { phytosterol }\end{array}$ & $\begin{array}{l}\text { Ananas comosus, Ficus racemosa, Ficus religiosa, Eupatorium triplinerve, Eclipta alba,, Amorphophallus campanulatus, } \\
\text { Euphorbia prostrate, Acacia sumo, Smilex china, Azadirachta indica, Ochrocarpus longifolius, Tribulus terrestris }\end{array}$ \\
\hline B & Campesterol & $\begin{array}{l}\text { Aerva lonata, Linum usitatissimum, Polyalthia longifolia, Ochrocarpus longifolius, Alangium salvifolium, Paspalum } \\
\text { scrobiculatum, Ochrocarpus longifolius, tribulus terrestris }\end{array}$ \\
\hline C & Beta-sitosterol & $\begin{array}{c}\text { Terminalia arjuna, Saraca asoca, Clerodendrum phlomidis, Gmelia arborea, Boerhavia diffusa, Piper longum, Ficus } \\
\text { glomerata, Abies webbiana, Curcuma longa, Aloe vera, Asparagus racemosus, Cyperus rotundus, Piper retrofroctum, } \\
\text { Polyalthia longifolia, Sismbrium irio, Raphanus sativus, Gynandropsis gynanndra, Althoea officinalis, Grewia tenax, Linum } \\
\text { usitatissimum, Ailanthus excels, Boswellia serrata, Vitis vinifera, Crotalaria verrucosa, Ficus rumphii, Cissus quadrangularis, } \\
\text { Blumea lacera, Nyctanthes arbortristis, Cordia dichotoma, Barleria prionitis, Ficus bengalensis, Ficus religiosa, Ficus lacor, } \\
\text { Ficus hispida, Albizzia lebbeck, Terminalia bellerica, Benincasa hispida, Centella asiatica, Anethum sowa, Foenieulum } \\
\text { vulgare, Inula racemosa, Plumbago zeylanica, Wrightia tinctoria, Nerium indicum, Calotropis procera, Hemidesmus indicus, } \\
\text { Swertia chirayita, Convolvulus pluricaulis, Solanum indicum, Datura metel, Bacopa monnieri, Oroxylum indicum, Adhatoda } \\
\text { vasica, Vitex negundo, Aegle marmelos, Azadirachta indica, Aconitum heterophylum, Tinospora cordifolia, Crataeva } \\
\text { nurvala, Mesua ferrea, Shorea robusta }\end{array}$ \\
\hline
\end{tabular}




\begin{tabular}{|c|c|c|}
\hline 4 & & Flavanoids \\
\hline A & Flavanoids- flavone, Isoflavanoids & $\begin{array}{c}\text { Aerva lanata, Asparagus racemosa, Ficus bengalensis, Cassia angustifolia, Cissampelos pareira, Swertia chirata, } \\
\text { Pongamia pinnata, Thespesia populnea,, Salvadora persica, Ougeinia oojeinensis, Leucas cephalotes, Cynodon dactylon, } \\
\text { Juniperus communis, Pinus roxburghii, Dallbergia sissoo, Oroxylum indicum, Cedrus deodara, Butea monosperma, Allium } \\
\text { sativum, Taraxacum officinale, Phaseolus trilobus, Cassia absus, Nymphea alba, Annona sqquamosa, Madhuca longifolia, } \\
\text { Argyreia speciosa, Hordeum vulgare, Pluchea lanceolata, Dolichos biflorus, Luffa echinata, Nymphea alba, Cleome } \\
\text { gynandra, Premna integrifolia, Hordeum vulgare }\end{array}$ \\
\hline B & Luteolin & $\begin{array}{c}\text { Luffa echinata, Phaseolus trilobus, Nymphea alba, Cleome gynandra, Premna integrifolia, Hordeum vulgare, Hydnocarpus } \\
\text { wightiana, Ferula northax, Apiu graveolans, }\end{array}$ \\
\hline C & Genistein & Prunus puddum, Dolichos biflorus \\
\hline D & Quercetin & $\begin{array}{c}\text { Moringa oleifera, Allium sativum, Taraxacum officinale, Phaseolus trilobus, Cassia absus, Clitoria ternatea, Nymphea alba, } \\
\text { Annona sqquamosa, Madhuca longifolia, Dendrophthoe falcate, Argyreia speciosa, Hordeum vulgare, Pluchea lanceolata, } \\
\text { Prunus avium, Typha elephantine, Calycopteris floribunda, Typha australis, Crataeva nurvala, Saraka asoca, Syzygium } \\
\text { cumini }\end{array}$ \\
\hline $\mathbf{E}$ & Epigallo-catechin gallate & Green tea \\
\hline 5 & Piperine & Piper cubeba, Piper chaba, Piper longum, Piper nigrum \\
\hline 6 & Diallyl sulphide & Allium sativum \\
\hline 7 & Gingerol & Zingiber officinale \\
\hline 8 & Crocetin & Saffron crocus, Crocus sativus \\
\hline 9 & Curcumin(diferuloylmethane) & Curcuma longa \\
\hline 10 & Indole-3-carbinol & Cruciferae family plants \\
\hline 11 & Lycopene & Tomatoes, Red carrots, Watermelons, Pink grapefruit, Papayas, Guavas \\
\hline 12 & Rosmarinic acid & Ocimum species- Ocimum sanctum, Ocimu Basilicum \\
\hline 13 & Fisetin & Onion, Cucumber \\
\hline 14 & Resveratrol & $\begin{array}{c}\text { Syzygium cumini, Artocarpus heterophyllus, Morus rubra, Vitis vinifera, Aegle marmelos, Annona squamosa, Emblica } \\
\text { officinalis, Hippophae rhamnoided, Tamarindus indiaca, Morus rubra, Syzygium cumini, Artocarpus heterophyllus, Punica } \\
\text { granatum }\end{array}$ \\
\hline
\end{tabular}

Table 2: Phytochemicals present in medicinal plants.

therapeutics along with current chemotherapy drugs against various types of cancer. Although a number of other phytochemicals could be also added to this list, it is better to remain until more clinical trials support their anticancer effect (Table 2).

\section{Conclusion}

In literary review, the potential anticancer phytochemicals and dietary agents, their molecular targets, and their mechanisms of actions have been discussed. The understanding of molecular mechanism of a specific plant derived compound against a particular type of cancer will lead to the invention of novel drug and drug targets for therapeutic intervention. Therefore, for many of phytochemicals, it is too early to draw conclusion for their anticancer actions. Moreover, much remains to be learned about pharmacokinetics, drug interactions, ideal dosages, long-term safety and adverse effects of phytochemicals proposed for cancer treatment. On the other hand, fortunately molecular mechanisms responsible for anticancer effect of several medicinal plants have been revealed by in vitro studies. These mechanisms include antioxidation, carcinogen inactivation, anti-proliferation, cell cycle arrest, induction of apoptosis, and inhibition of angiogenesis or a combination of these mechanisms. It is believed that herbal preparations containing multiple phytochemicals may have greater effects than the same phytochemical taken separately. Based on this belief, combinations of the anticancer phytochemicals may have more effect and yield more potent therapeutic agents for cancer.

\section{References}

1. Dashwood RH, Arbogast DN, Fong AT, Pereira C (1989) Quantitative interrelationships between aflatoxin B1 carcinogen dose, indole-3-carbinol anticarcinogen dose, target organ DNA adduction and final tumor response. Carcinogenesis 10: 17581.
2. Bajpai M, Pande A, Tewari SK, Prakash D (2005) Phenolic contents and antioxidant activity of some food and medicinal plants. Int J Food Sci Nutr 56: 287-291.

3. Black HS, Mathews-Roth MM (1991) Protective role of butylated hydroxytolune and certain carotenoids in photocarcinogenesis. Phytochem Photobiol 53:707716 .

4. Liu RH (2004) Potential synergy of phytochemicals in cancer prevention mechanism of action. J Nutr 134: 3479S-3485S.

5. Ali H, Dixit S, Ali D, Alqahtani S, Alkahtani S, et al. (2015) Isolation and evaluation of anticancer efficacy of stigmasterol in a mouse model of DMBAinduced skin carcinoma. Drug Design, Development and Therapy, Dove Press 9: 2793-2800.

6. Birt DF, Hendrich S, Wang W (2001) Dietary agents in cancer prevention flavonoids and isoflavonoids. Pharmacol Ther 90: 157-177.

7. Kumar N, Shibata D, Helm J, Coppola D, Malafa M (2007) Green tea polyphenols in the prevention of colon cancer. Front Biosci 12: 2309-2315

8. Alinejad B, Ghorbani A, Sadeghnia HR (2013) Effects of combinations of curcumin, linalool, rutin, safranal, and thymoquinone on glucose/serum deprivation-induced cell death. Avicenna J Phytomed 3: 321-328.

9. Athar M, Back JH, Tang X, Kim KH, Kopelovich L, et al. (2007) Resveratrol a review of preclinical studies for human cancer prevention. Toxicol Appl Pharmacol 224: 274-283.

10. Hosseini A, Ghorbani A (2015) Cancer therapy with phytochemicals: evidence from clinical studies. Avicenna J Phytomed 5: 84-97.

11. Le Bon AM, Siess MH (2000) Organosulfur compounds from allium and the chemoprevention of cancer. Drug Metabol Drug Interact 17: 51-79.

12. Sastry JL, Vijnana D (2015) Chaukhambha orientalia. Volume II. Varanshi, Reprint Edition.

13. Ministry of Health and Family Welfare (2008) Department of AYUSH Government of India, New Delhi, India. API. Part I. Volumes 1-6, 2008. 